\title{
Update in Tuberculosis and Non-Tuberculous Mycobacteria 2017
}

\section{Authors:}

Ibrahim Abubakar(1), Rishi K. Gupta(1), Molebogeng X. Rangaka(1), Marc Lipman(2,3)

Institutions:

1. Institute for Global Health, University College London, London, UK

2. UCL-TB and UCL Respiratory, University College London, London, UK

3. Royal Free London NHS Foundation Trust, London, UK

Corresponding author: Ibrahim Abubakar (i.abubakar@ucl.ac.uk)

Words: 3654

References: 48 


\section{Introduction}

Tuberculosis (TB) caused an estimated 10.4 million cases and 1.7 million deaths in 2016 (1). While global TB incidence and mortality are falling by 2 and 3\% per year respectively, these declines are far behind what is required to achieve the World Health Organization (WHO) End TB Strategy targets, which aims for $90 \%$ and $95 \%$ reductions in TB incidence and mortality between 2015-2035, respectively(2). Consequently, major advances in our understanding of TB pathogenesis (including vaccine development) and transmission, along with new tools for the diagnosis and treatment of both active TB disease and latent infection are needed. Further, the global importance of non-tuberculous mycobacterial (NTM) disease is being increasingly recognised as incidence rises $(3,4)$. This review summarises the advances in both TB and NTM published in the Journal and other journals in 2017.

\section{Immunology of TB}

The immunological mechanisms underlying TB pathogenesis including susceptibility to infection, progression to disease, recurrence and response to vaccination remain poorly understood. Four studies in the Journal provide new insight into TB pathogenesis, with consequences for vaccination and TB control.

The initiation of adaptive immune responses is an integral aspect of the host response to TB. Shah and colleagues investigated the mechanism of action of toll-interacting protein (TOLLIP), an innate immune response regulator known to be associated with $\mathrm{TB}(5)$. Using cellular immunology approaches, they examined monocyte mRNA expression and TB-specific immune functions, along with the association between TOLLIP variation and BCG-induced T-cell responses and latent TB susceptibility. The authors report a functional TOLLIP promoter region polymorphism associated with decreased mRNA expression and BCG-specific memory T-cell responses, plus increased TB susceptibility with the TOLLIP-deficient genotype. This was despite there being an increased inflammatory response and decreased mycobacterial replication. The authors postulate that TOLLIP may be a vaccine adjuvant or host-directed therapy target worth further investigation that might enhance immunity and minimise adverse cytokine response.

Leu and colleagues investigated the role of SP110b (a nuclear protein) in host innate immunity to $M$. tuberculosis infection(6). Using cellular approaches and mouse models, they showed that SP110b downregulates TNF- $\alpha$ production of interferon gamma active cells, preventing apoptosis. Furthermore, Ipr1, which is a homologue of SP110b, impairs TNF- $\alpha$ production stopping further pulmonary necrosis. They concluded that, by modulating nuclear factor $\mathrm{kB}(\mathrm{NF}-\mathrm{kB})$ activity, this 
protein has a significant role in the inflammatory response that protects against TB and may also be a target for future host-directed therapies.

Scriba and colleagues investigated the effects of previous TB on immunity by assessing whether previous disease leads to a less effective immune response to future TB exposure, thereby increasing the risk of recurrence(7). They hypothesized that prior TB disease leads to differences between latently infected individuals in the repertoire of M. tuberculosis-derived epitopes recognized by $\mathrm{T}$ cells. By assessing $\mathrm{T}$ cell responses in adolescents and adults, they identified two sets of $M$. tuberculosis epitopes, with contrasting immune reactivity in latently infected individuals distinguished by previous disease status. This was seen only in type 2 specific T-cell epitopes, which were less likely to be recognised by those with previous TB in the preceding 6 years compared to people with no history of TB. The sets of type $2 \mathrm{~T}$-cell epitopes were homologous to human microbiome bacterial sequences. Furthermore, persistence of the type 1 responses and loss of the type 2 response after antiTB treatment was noted. The authors concluded that there may be persistence of antigens that affect type $1 \mathrm{~T}$-cell epitopes after treatment and/or that type 2-specific responses may be induced by crossreactive microbiome-derived antigens. Assuming these results are not due to re-exposure and reinfection in this high TB burden setting, the findings may have implications for vaccine development, as the persistence of antigens could influence the subsequent efficacy of BCG and new vaccines (8).

Immunisation of individuals already infected with M. tuberculosis with BCG or killed bacilli may lead to Koch-type reactions (including a necrotic reaction at the site of injection leading to ulceration). Van Zyl-Smit and colleagues assessed whether a candidate vaccine (AERAS-402 adenovirus serotype 35 replication-deficient vaccine), which contains DNA encoding for mycobacterial antigens 85A, $85 \mathrm{~B}$, and TB10.4, is a cause of pulmonary or systemic adverse effects in individuals with current or previously treated $\mathrm{TB}(9)$. All 36 participants with active TB and most (35 individuals; 97\%) individuals with previously treated active TB experienced at least one adverse effect, though these were not deemed to be clinically significant. The authors concluded that there was a robust immune response based on the proportion of T-cells producing specific cytokines and, in these populations, there was no evidence of Koch phenomenon-type reactions attributable to the vaccine.

\section{Diagnosis and treatment of active TB disease}

In the Journal, Lessells et al reported the results of a cluster randomised-controlled trial examining the impact of point-of-care compared to hospital laboratory implementation of Xpert MTB/RIF, a molecular test for TB and rifampicin resistance(10), on TB treatment initiation among individuals who were either HIV positive (92.5\%), and/or had risk-factors for MDR-TB (45\%)(11). There was no difference between the point-of-care and laboratory arms in the primary outcome of the proportion of 
individuals with culture-confirmed pulmonary TB who had initiated appropriate treatment at 30 days, though the median time to treatment initiation was shorter in the point-of-care arm ( 1 day vs. 7 days). The authors' power to detect a difference in their primary outcome was limited by an impressive $93 \%$ of individuals with Xpert-positive/rifampicin-sensitive disease initiating appropriate therapy within 30 days in the laboratory arm. Most of the delays in treatment initiation beyond 30 days in both arms were in individuals with Xpert-negative, or Xpert-positive/rifampicin-resistant disease. This highlights a need for the development of new point-of-care diagnostics with improved sensitivity, and improved access to second-line drug-resistance testing and treatment services, which may reduce delays in treatment initiation further.

The WHO has recently endorsed the new Xpert Ultra assay as an alternative to Xpert MTB/RIF(12). Recent evaluations of Xpert Ultra suggest that it offers improved sensitivity compared to Xpert MTB/RIF in smear-negative TB (63\% vs. 46\%), HIV-associated pulmonary TB (90\% vs. $77 \%)$ and HIV-associated TB meningitis (95 vs. 45\%)(13-15). However, the improved sensitivity may be associated with a modest reduction in specificity, particularly in individuals with a recent history of TB and in high TB-incidence settings, which requires further evaluation(13). A further cartridge has been developed for the GeneXpert platform which detects mutations conferring resistance to fluoroquinolones, aminoglycosides, and isoniazid(16). Evaluation of the assay in China and Korea has demonstrated promising diagnostic accuracy, suggesting that it could have a role as a point-of-care test in settings with high prevalence of MDR-TB(17), although further evaluation in other settings is needed.

A national, routine whole-genome sequencing (WGS) service for mycobacterial infections was recently launched in the UK(18). This has the potential to offer prompt, reproducible drug-resistance profiles to clinicians, and may ultimately replace the need for phenotypic drug-susceptibility testing. However, the impact of WGS is limited by the need for mycobacterial culture prior to sequencing, though direct WGS from sputum samples is now reported(19). Universal WGS could enable almost real-time reconstruction of phylogenetic relationships between isolates, and may facilitate identification of clusters, and inference of transmission, with greater than current resolution. An issue for WGS is the increasing evidence demonstrating the degree of within-host diversity of M.tuberculosis strains. In the Journal, Metcalfe et al. demonstrated the potential role of minority mycobacterial subpopulations in contributing to phenotypic drug-resistance(20). The authors used a targeted, high-coverage next generation sequencing technique (single molecule overlapping reads; SMOR) to resolve discordance between phenotypic drug-resistant isolates without corresponding Sanger sequencing-detected genotypic resistance variants. They found that minor resistant variants 
could be identified using SMOR in $80 \%$ of these discordant isolates. This supports the view that micro-evolution leading to within-host diversity of M.tuberculosis may influence drug-susceptibility; though the precise role of these subpopulations in the evolution of acquired drug-resistance requires further evaluation $(21,22)$.

Advances in TB treatment in 2017 have included release of the preliminary results of the Standardised Treatment Regimen of Anti-TB Drugs for Patients with MDR-TB (STREAM) Trial at The Union World Conference on Lung Health in Mexico(23). This international, multi-centre trial is comparing the standard 20-month regimen recommended by the 2011 WHO guidelines with the WHOendorsed(24) 9-month regimen consisting of moxifloxacin, clofazimine, ethambutol and pyrazinamide given for nine months, supplemented by kanamycin, isoniazid and prothionamide in the first four months, among patients with MDR-TB, without evidence of resistance to fluoroquinolones or injectables(25). The preliminary results demonstrate similar efficacy, though non-inferiority to standard therapy was not formally demonstrated. Final published results are pending (23). However, there is recognition that, in some settings, only a minority of MDR-TB cases will be eligible for the 9month regimen due to resistance to second-line drugs (estimated as $4.2-11.1 \%$ in three European studies)(26-28). This highlights an ongoing need for new antimicrobial regimens for MDR-TB, and also for host-directed therapies to improve treatment outcomes(29). In the Journal, Ganmaa et al conducted a randomised-controlled trial to assess the impact of high-dose vitamin D3 on response to anti-microbial therapy in adults with drug sensitive pulmonary TB in Mongolia, with the primary outcome of time to sputum culture conversion(30). The authors found that supplementation did not reduce time to culture conversion overall, though significant effect modification was noted in individuals with genetic variants in the vitamin D signalling pathway. This supports previous evidence(31-33); and together, the results suggest that we should now move on from investigating whether vitamin D can improve the outcome of treatment for TB disease, when given with standard TB therapy.

\section{Diagnosis of latent tuberculosis infection}

In 2017 the diagnosis of latent TB infection (LTBI) and the risk of progressive TB based on interferon gamma release assay (IGRA) results continued to be important themes in the Journal. Conversion of a QuantiFERON-TB (QFT) IGRA from a negative to positive test likely signals M.tuberculosis infection and carries $>2$-fold risk of progression to TB in untreated individuals(34). However, variation in the definitions of IGRA conversions and reversions can affect the utility of the assay in the programmatic management of LTBI. 
Using a combined cohort approach Nemes and colleagues conducted an analysis of retrospective data to derive a stringent risk-validated definition of QFT conversion to improve the consistency of serial testing algorithms(35). They used data from multiple HIV-negative populations tested with IGRA, which included 5,357 healthy adolescents from a high-risk setting, M.tuberculosis-unexposed individuals from a low-burden setting (controls), and patients with definite pulmonary tuberculosis. Cohorts were followed for at least two years. Inter-assay variability was assessed and distributions of IFN- $\gamma$ response levels analyzed and correlated with the risk of TB. The authors proposed an internally validated risk strata of IFN- $\gamma$ values $<0.2 \mathrm{IU} / \mathrm{ml}, 0.2-0.34 \mathrm{IU} / \mathrm{ml}, 0.35-0.7 \mathrm{IU} / \mathrm{ml}$, and $>0.7 \mathrm{IU} / \mathrm{ml}$ (TST positivity trend was $15 \%, 53 \%, 66 \%$, and $91 \%$, respectively). Individuals with values less than $0.2 \mathrm{IU} / \mathrm{ml}$ are presumed 'true negatives' and those with values greater than $0.7 \mathrm{IU} / \mathrm{ml}$ 'true positives' with established M.tuberculosis infection. As expected, all M.tuberculosis-unexposed individuals had IFN- $\gamma$ values $<0.2 \mathrm{IU} / \mathrm{ml}$, and the majority ( $87 \%$ ) of patients with active tuberculosis had IFN- $\gamma$ values $>0.7 \mathrm{IU} / \mathrm{ml}$. The authors suggest a strict QFT conversion definition of IFN- $\gamma$ value $<0.2$ to $>0.7$ would improve detection of those likely to progress; converters had a 10 -fold higher risk of TB than nonconverters (defined as maintaining values $<0.2 \mathrm{IU} / \mathrm{ml}$ over 2 years). Conversions and reversions tend to be around the manufacturer's suggested threshold $(0.35 \mathrm{IU} / \mathrm{ml})$. The authors thus propose the introduction of a zone of uncertainty for QFT (between $0.2 \mathrm{IU} / \mathrm{ml}$ and $0.7 \mathrm{IU} / \mathrm{ml}$ ), as is done when interpreting T-Spot.TB results. Interestingly, individuals with at least one conversion in this zone of uncertainty were shown to have a statistically similar risk of TB as non-converters. Weak immune responses as well as issues affecting assay reproducibility may contribute to conversions in this zone. It will be important to determine the implications of conversion values in the uncertainty zone with respect to clinical utility, the timing of re-testing, and TB risk in further independent cohorts(36). Definitions proposed in this paper would need external validation especially in HIV-positive individuals and in individuals with other immunosuppressive conditions.

\section{Transmission of TB}

Exploring heterogeneity in the transmission dynamics of drug sensitive and drug resistant TB in communities can offer a powerful strategy for strengthening TB control(37). Shrestha and colleagues demonstrated the utility of this approach using United States (US) data(38).

TB incidence in the US has remained low but steady at approximately 3.0 per 100,000 population since 2013. The changing demographic makeup or a plateau in the effect of currently implemented interventions may partly explain this. Foreign-born individuals account for two-thirds of new TB 
cases, with a TB incidence of 15.1 per 100,000(39). Shrestha and colleagues characterized drivers and dynamics of TB in the relatively high-burden states of California, Florida, New York, and Texas to examine state-to-state heterogeneity in TB risk and assess whether the declines in TB incidence would continue in the absence of additional interventions or resources. They developed an individual-based model of TB calibrated to state-specific demographics and TB incidence data stratified by age and foreign-born status. They then used this to infer differences in the natural history of TB and conduct state-specific 10-year projections of TB from 2015. They found that the size and composition of the foreign-born population and TB transmission dynamics contribute to substantial state-to-state heterogeneity. Without additional resources or further state-specific intervention, the model projected a plateauing of the decline in TB incidence by 2025 in all four states, suggesting that these US states would likely not meet the target of achieving pre-elimination (incidence $<1 / 100,000$ ), and moving towards elimination by 2035 .

Genetic variations in mycobacterial strains can provide novel insights to understanding TB transmission but are less frequently studied than host factors. Two studies in the Journal offered advances in this field. Nebenzahl-Guimares and colleagues used a tuberculosis registry and strain collection in the Netherlands to identify mycobacterial genetic markers associated with increased transmissibility, and to examine whether these lead to altered in vitro immune responses(40). Controlling for host factors for clustering, a set of 100 drug-sensitive M. tuberculosis strains either least or most likely to be transmitted, based on their clustering patterns, were identified a priori and analyzed using WGS and evolutionary convergence approaches. In vitro cytokine production and neutrophil responses to a subset of the strains with or without the identified mutations associated with increased transmissibility were also measured. The authors hypothesized that clustered strains have consistent genetic differences compared with unclustered ones, and that the genes or intergenic regions associated with the difference affect the host immune response. The loci espE, PE-PGRS56, Rv0197, Rv2813-2814c, and Rv2815-2816c were identified as targets of convergent evolution among transmissible strains. Four of these regions were subsequently confirmed in an independent strain cohort and functionally validated to demonstrate that mutations in the targets affected in vitro monocyte and T-cell cytokine production, neutrophil reactive oxygen species release, and apoptosis. The 100 strain size used in this study, derived from patients with drug sensitive TB originating from 44 different countries, is small. The findings need to be validated in larger cohorts of longitudinally collected strains from both high- and low-burden settings to further understand their epidemiological and clinical significance in TB transmission. 
Molecular diagnostic tools that can rapidly confirm the presence of TB infection and identify the full range of resistance mutations to anti-tuberculosis drugs would revolutionize case detection and curtail transmission. The study by Farhat et al partially addresses this challenge $(41)$. Using a large $(\mathrm{N}=1,738)$ geographically diverse, prospectively collected archive of clinical isolates of drug resistant (DR) TB, the authors aimed to identify molecular determinants of resistance to 13 anti-TB drugs using molecular inversion probes, and present a validated prediction model for diagnosing resistance to first- and second-line drugs based on the detection of mutations within the full length of 28 presumed DR loci. The main finding was that predicted resistance to rifampicin and isoniazid, the only fully characterized drugs, exceeded $90 \%$ sensitivity and specificity but was lower for the other drugs or could not reliably be determined. The number of mutations needed to diagnose resistance was large (238 across 18 genetic loci for the 13 drugs). This presents a challenge for future diagnostic tools, which will have to allow for a high dimension of mutation detection. This can be possible with WGS which would also allow identification of previously undetected or unknown DR loci that code resistance to one or more drugs and thus improve the sensitivity of anti-TB drugs other than rifampicin and isoniazid. This study illustrates the importance of pooling data across laboratories and geographic regions to aid the development of predictive models for DR-TB with greater accuracy for detecting resistance mutations.

\section{Non-tuberculous mycobacteria}

Pulmonary NTM infections are increasingly common, and difficult to successfully treat $(4,42)$. Recent work using a population-based cohort of patients with NTM respiratory isolates in Oregon, USA has highlighted the appreciable mortality ( $>35 \%$ at 5 years) - which was often due to patients' co-existent illnesses(43). However, significant immunocompromise (such as advanced HIV infection) or extensive structural lung disease (for example in Cystic Fibrosis) now accounts for a minority of NTM cases. In the US, chronic pulmonary NTM infection and disease are seen increasingly among people with apparently normal (or near-normal) immunity and minimal initial lung damage(4). Reasons for this, along with new approaches to treat pulmonary NTM, are the subject of two 2017 Journal publications. These areas were identified by a NTM Research Consortium Workshop as some of the main priorities for patient-centred research clinical and translational science (44).

Familial clustering of pulmonary NTM disease is rare but has been reported in carefully-performed studies(45). Applying whole-exome sequencing technology to 9 families with pulmonary NTM, and then including a further 57 sporadic cases (where the unaffected family members acted as controls for all tested cases), Chen and colleagues identified a 20-cM (20,000 kilobase) region on chromosome 6q12-6q16 with a HLOD (heterogeneity logarithm of the odds) score of 3.9 ( $>3.3$ was regarded as indicating linkage)(46). This fitted best under a recessive disease model with $100 \%$ penetrance and a 
risk allele frequency of 5\%. Using gene-level linkage analysis, they determined the genes potentially driving the linkage signals. TTK protein kinase (TTK) gene, which has a role in regulating proper chromosomal alignment and hence controlling DNA damage and repair, was most strongly associated(46). It remains to be seen whether this proposed mechanism (where aberrant TTK gene function could result in failure of local innate pulmonary immunity and so allow environmental NTM to colonise, infect and ultimately cause disease) is relevant to many NTM patients with unexplained disease, or if it is a phenomenon arising in patients selected by their atypical - and hence who were studied. Further work will help to define and characterise the extent of this and other gene defects in NTM infected populations.

The publication of a randomised, controlled trial of liposomal amikacin for inhalation (LAI)(47) is an important step in approaches to treatment which, unlike many other therapies for NTM, may be both effective and reasonably tolerated (48). In this study, 89 adults (of 136 screened) with treatmentrefractory pulmonary NTM received either once daily LAI or liposomal placebo. The primary endpoint was change in sputum mycobacterial growth after 12 weeks using a semi-quantitative scale (SQS). This efficacy measure represented transitions between grades of growth on either solid media (with a set range of colony-forming units being graded as different categorical values) or a change from positive to negative in liquid culture. Time to positivity in liquid medium (as a surrogate of mycobacterial load) was not used. Prior to study enrolment, patients had persistently positive sputum cultures, despite in many cases at least 12-months of continuous therapy for NTM. Patients were designated as either having predominately M. avium complex (two-thirds of subjects) or predominant M. abscessus infection (one-third). The majority of subjects had cavitatory NTM disease; and approximately one in five of participants had underlying CF.

The trial showed no difference in SQS between the two arms, though improvements were found in the secondary endpoint of the proportion achieving sputum conversion (in particular for patients with predominantly $M$. avium complex) and also the tertiary endpoint of change in 6-minute walk distance. Interestingly health status measures such as St. George's Respiratory Questionnaire and a CF-adapted and non-CF NTM specific questionnaire did not change. This may be explained by dysphonia, cough and infectious exacerbations of respiratory disease being more common within the LAI arm - all of which would reduce reported health status. The latter is a concern, given that Amikacin might be expected to prevent some respiratory infections. However, it is unclear which (if any) organisms were isolated from sputum during the acute deteriorations. It is important to note LAI was no more likely to result in audiological changes or impaired renal function than placebo. The one death within the LAI arm was felt not to be due to NTM disease or drug treatment. The reasonable tolerability of LAI enables us to hope that when used for longer durations it will continue to offer a similar reasonable safety profile. 
One issue is whether the addition of a single drug to a potentially failing infection management regimen is wise. In post-hoc analysis, individuals who did not respond to LAI had either baseline amikacin-resistance within their isolates, or developed this subsequently on treatment. $10 \%(9 / 89)$ patients had baseline resistance to amikacin, whilst a further $6 \%(5 / 89)$ were resistant at week 12 or 24 of therapy. This information is helpful in determining who is likely to respond to (and therefore most benefit from) LAI. It also has implications for clinicians who may ask if the drug can be of greater benefit given earlier in therapy.

\section{Conclusions}

Research published in the Journal and elsewhere in 2017 has improved our knowledge of tuberculosis pathogenesis, transmission and the management of latent and active tuberculosis. Similarly, new work provides further promising insights regarding NTM. These contributions are critical if we are to achieve the global plan to end tuberculosis; though there remains much to do. 


\section{References}

1. World Health Organization. Global Tuberculosis Report 2017. 2017; at $<\mathrm{http} / / /$ www.who.int/tb/publications/global_report/en/>.

2. World Health Organization. The End TB Strategy. 2015; at $<\mathrm{http}: / /$ www.who.int/tb/strategy/End_TB_Strategy.pdf?ua=1>.

3. Brode SK, Daley CL, Marras TK. The epidemiologic relationship between tuberculosis and non-tuberculous mycobacterial disease: a systematic review. Int J Tuberc Lung Dis 2014;18:1370-1377.

4. Adjemian J, Olivier KN, Seitz AE, Holland SM, Prevots DR. Prevalence of nontuberculous mycobacterial lung disease in U.S. Medicare beneficiaries. Am J Respir Crit Care Med 2012;185:881-6.

5. Shah JA, Musvosvi M, Shey M, Horne DJ, Wells RD, Peterson GJ, Cox JS, Daya M, Hoal EG, Lin L, Gottardo R, Hanekom WA, Scriba TJ, Hatherill M, Hawn TR. A Functional TollInteracting Protein Variant Is Associated with Bacillus Calmette-Guérin-Specific Immune Responses and Tuberculosis. Am J Respir Crit Care Med 2017;196:502-511.

6. Leu J-S, Chen M-L, Chang S-Y, Yu S-L, Lin C-W, Wang H, Chen W-C, Chang C-H, Wang JY, Lee L-N, Yu C-J, Kramnik I, Yan B-S. SP110b Controls Host Immunity and Susceptibility to Tuberculosis. Am J Respir Crit Care Med 2017;195:369-382.

7. Scriba TJ, Carpenter C, Pro SC, Sidney J, Musvosvi M, Rozot V, Seumois G, Rosales SL, Vijayanand P, Goletti D, Makgotlho E, Hanekom W, Hatherill M, Peters B, Sette A, Arlehamn CSL. Differential Recognition of Mycobacterium tuberculosis -Specific Epitopes as a Function of Tuberculosis Disease History. Am J Respir Crit Care Med 2017;196:772-781.

8. Mangtani P, Abubakar I, Ariti C, Beynon R, Pimpin L, Fine PEM, Rodrigues LC, Smith PG, Lipman M, Whiting PF, Sterne JA. Protection by BCG Vaccine Against Tuberculosis: A Systematic Review of Randomized Controlled Trials. Clin Infect Dis 2014;58:470-480.

9. van Zyl-Smit RN, Esmail A, Bateman ME, Dawson R, Goldin J, van Rikxoort E, Douoguih M, Pau MG, Sadoff JC, McClain JB, Snowden MA, Benko J, Hokey DA, Rutkowski KT, Graves A, Shepherd B, Ishmukhamedov S, Kagina BMN, Abel B, Hanekom WA, Scriba TJ, Bateman ED. Safety and Immunogenicity of Adenovirus 35 Tuberculosis Vaccine Candidate in Adults with Active or Previous Tuberculosis. A Randomized Trial. Am J Respir Crit Care Med 2017;195:1171-1180. 
10. World Health Organization. Xpert MTB/RIF assay for the diagnosis of pulmonary and extrapulmonary TB in adults and children. 2013; at

$<$ http://apps.who.int/iris/bitstream/10665/112472/1/9789241506335_eng.pdf?ua=1>.

11. Lessells RJ, Cooke GS, McGrath N, Nicol MP, Newell ML, Godfrey-Faussett P. Impact of point-of-care xpert MTB/RIF on tuberculosis treatment initiation: A cluster-randomized trial. Am J Respir Crit Care Med 2017;196:901-910.

12. World Health Organization. WHO Meeting Report of a Technical Expert Consultation: Noninferiority analysis of Xpert MTB/RIF Ultra compared to Xpert MTB/RIF. at $<$ http://apps.who.int/iris/bitstream/10665/254792/1/WHO-HTM-TB-2017.04-eng.pdf?ua=1>.

13. Dorman SE, Schumacher SG, Alland D, Nabeta P, Armstrong DT, King B, Hall SL, Chakravorty S, Cirillo DM, Tukvadze N, Bablishvili N, Stevens W, Scott L, Rodrigues C, Kazi MI, Joloba M, Nakiyingi L, Nicol MP, Ghebrekristos Y, Anyango I, Murithi W, Dietze R, Lyrio Peres R, Skrahina A, Auchynka V, Chopra KK, Hanif M, Liu X, Yuan X, et al. Xpert MTB/RIF Ultra for detection of Mycobacterium tuberculosis and rifampicin resistance: a prospective multicentre diagnostic accuracy study. Lancet Infect Dis 2017;18:76-84.

14. Chakravorty S, Simmons AM, Rowneki M, Parmar H, Cao Y, Ryan J, Banada PP, Deshpande S, Shenai S, Gall A, Glass J, Krieswirth B, Schumacher SG, Nabeta P, Tukvadze N, Rodrigues C, Skrahina A, Tagliani E, Cirillo DM, Davidow A, Denkinger CM, Persing D, Kwiatkowski R, Jones M, Alland D. The New Xpert MTB/RIF Ultra: Improving Detection of Mycobacterium tuberculosis and Resistance to Rifampin in an Assay Suitable for Point-ofCare Testing. In: Nacy CA, editor. MBio 2017;8:e00812-17.

15. Bahr NC, Nuwagira E, Evans EE, Cresswell F V, Bystrom P V, Byamukama A, Bridge SC, Bangdiwala AS, Meya DB, Denkinger CM, Muzoora C, Boulware DR, Williams DA, Taseera K, Nyehangane D, Ivan M, Orikiriza P, Rhein J, Hullsiek KH, Musubire A, Pastick K, Nabeta P, Mwesigye J, Rajasingham R. Diagnostic accuracy of Xpert MTB/RIF Ultra for tuberculous meningitis in HIV-infected adults: a prospective cohort study. Lancet Infect Dis 2018;18:6875.

16. Chakravorty S, Roh SS, Glass J, Smith LE, Simmons AM, Lund K, Lokhov S, Liu X, Xu P, Zhang G, Via LE, Shen Q, Ruan X, Yuan X, Zhu HZ, Viazovkina E, Shenai S, Rowneki M, Lee JS, Barry CE, Gao Q, Persing D, Kwiatkawoski R, Jones M, Gall A, Alland D. Detection of Isoniazid-, Fluoroquinolone-, Amikacin-, and Kanamycin-Resistant Tuberculosis in an Automated, Multiplexed 10-Color Assay Suitable for Point-of-Care Use. In: Land GA, editor. J Clin Microbiol 2017;55:183-198.

17. Xie YL, Chakravorty S, Armstrong DT, Hall SL, Via LE, Song T, Yuan X, Mo X, Zhu H, Xu 
P, Gao Q, Lee M, Lee J, Smith LE, Chen RY, Joh JS, Cho Y, Liu X, Ruan X, Liang L, Dharan N, Cho S-N, Barry CE, Ellner JJ, Dorman SE, Alland D. Evaluation of a Rapid Molecular Drug-Susceptibility Test for Tuberculosis. N Engl J Med 2017;377:1043-1054.

18. Walker TM, Cruz ALG, Peto TE, Smith EG, Esmail H, Crook DW. Tuberculosis is changing. Lancet Infect Dis 2017;17:359-361.

19. Votintseva AA, Bradley P, Pankhurst L, del Ojo Elias C, Loose M, Nilgiriwala K, Chatterjee A, Smith EG, Sanderson N, Walker TM, Morgan MR, Wyllie DH, Walker AS, Peto TEA, Crook DW, Iqbal Z. Same-Day Diagnostic and Surveillance Data for Tuberculosis via WholeGenome Sequencing of Direct Respiratory Samples. In: Tang Y-W, editor. J Clin Microbiol 2017;55:1285-1298.

20. Metcalfe JZ, Streicher E, Theron G, Colman RE, Allender C, Lemmer D, Warren R, Engelthaler DM. Cryptic Microheteroresistance Explains Mycobacterium tuberculosis Phenotypic Resistance. Am J Respir Crit Care Med 2017;196:1191-1201.

21. Trauner A, Liu Q, Via LE, Liu X, Ruan X, Liang L, Shi H, Chen Y, Wang Z, Liang R, Zhang W, Wei W, Gao J, Sun G, Brites D, England K, Zhang G, Gagneux S, Barry CE, Gao Q. The within-host population dynamics of Mycobacterium tuberculosis vary with treatment efficacy. Genome Biol 2017;18:71.

22. Lieberman TD, Wilson D, Misra R, Xiong LL, Moodley P, Cohen T, Kishony R. Genomic diversity in autopsy samples reveals within-host dissemination of HIV-associated Mycobacterium tuberculosis. Nat Med 2016;22:1470-1474.

23. Preliminary results from STREAM trial provide insight into shorter treatment for multidrugresistant tuberculosis. at $<$ http://www.ctu.mrc.ac.uk/news/2017/preliminary_results_from_stream_trial_provide_insight _into_shorter_treatment_for_multidrug_resistant_tuberculosis>.

24. World Health Organization WH, Global Tuberculosis Programme. WHO treatment guidelines for drug-resistant tuberculosis : 2016 update. Who 2016;56.doi:WHO/HTM/TB/2016.04.

25. Nunn AJ, Rusen I, Van Deun A, Torrea G, Phillips PP, Chiang C-Y, Squire SB, Madan J, Meredith SK. Evaluation of a standardized treatment regimen of anti-tuberculosis drugs for patients with multi-drug-resistant tuberculosis (STREAM): study protocol for a randomized controlled trial. Trials 2014;15:353.

26. Balabanova Y, Fiebig L, Ignatyeva O, Riekstina V, Danilovits M, Jaama K, Davidaviciene E, Radiulyte B, Popa CM, Nikolayevskyy V, Drobniewski F. Multidrug-resistant TB in Eastern region of the EU: is the shorter regimen an exception or a rule? Thorax 2017;thoraxjnl-2016- 
209841.doi:10.1136/thoraxjnl-2016-209841.

27. van der Werf MJ, Hollo V, Ködmön C, Dara M, Catchpole M. Eligibility for shorter treatment of multidrug-resistant tuberculosis in the European Union. Eur Respir J 2017;49:1601992.

28. Lange C, Duarte R, Fréchet-Jachym M, Guenther G, Guglielmetti L, Olaru ID, Oliveira O, Rumetshofer R, Veziris N, van Leth F, European MDR-TB database collaboration *. Limited Benefit of the New Shorter Multidrug-Resistant Tuberculosis Regimen in Europe. Am J Respir Crit Care Med 2016;194:1029-1031.

29. Wallis RS, Maeurer M, Mwaba P, Chakaya J, Rustomjee R, Migliori GB, Marais B, Schito M, Churchyard G, Swaminathan S, Hoelscher M, Zumla A. Tuberculosis-advances in development of new drugs, treatment regimens, host-directed therapies, and biomarkers. Lancet Infect Dis 2016;16:e34-e46.

30. Ganmaa D, Munkhzul B, Fawzi W, Spiegelman D, Willett WC, Bayasgalan P, Baasansuren E, Buyankhishig B, Oyun-Erdene S, Jolliffe DA, Xenakis T, Bromage S, Bloom BR, Martineau AR. High-Dose Vitamin $\mathrm{D}_{3}$ during Tuberculosis Treatment in Mongolia. A Randomized Controlled Trial. Am J Respir Crit Care Med 2017;196:628-637.

31. Daley P, Jagannathan V, John KR, Sarojini J, Latha A, Vieth R, Suzana S, Jeyaseelan L, Christopher DJ, Smieja M, Mathai D. Adjunctive vitamin D for treatment of active tuberculosis in India: a randomised, double-blind, placebo-controlled trial. Lancet Infect Dis 2015; 15:528-534.

32. Martineau AR, Timms PM, Bothamley GH, Hanifa Y, Islam K, Claxton AP, Packe GE, Moore-Gillon JC, Darmalingam M, Davidson RN, Milburn HJ, Baker L V, Barker RD, Woodward NJ, Venton TR, Barnes KE, Mullett CJ, Coussens AK, Rutterford CM, Mein CA, Davies GR, Wilkinson RJ, Nikolayevskyy V, Drobniewski FA, Eldridge SM, Griffiths CJ. High-dose vitamin D3 during intensive-phase antimicrobial treatment of pulmonary tuberculosis: a double-blind randomised controlled trial. Lancet 2011;377:242-250.

33. Tukvadze N, Sanikidze E, Kipiani M, Hebbar G, Easley KA, Shenvi N, Kempker RR, Frediani JK, Mirtskhulava V, Alvarez JA, Lomtadze N, Vashakidze L, Hao L, Del Rio C, Tangpricha V, Blumberg HM, Ziegler TR. High-dose vitamin D3 in adults with pulmonary tuberculosis: a double-blind randomized controlled trial. Am J Clin Nutr 2015;102:1059-1069.

34. Machingaidze S, Verver S, Mulenga H, Abrahams DA, Hatherill M, Hanekom W, Hussey GD, Mahomed H. Predictive value of recent quantiFERON conversion for tuberculosis disease in adolescents. Am J Respir Crit Care Med 2012;186:1051-1056.

35. Nemes E, Rozot V, Geldenhuys H, Bilek N, Mabwe S, Abrahams D, Makhethe L, Erasmus M, 
Keyser A, Toefy A, Cloete Y, Ratangee F, Blauenfeldt T, Ruhwald M, Walzl G, Smith B, Loxton AG, Hanekom WA, Andrews JR, Lempicki MD, Ellis R, Ginsberg AM, Hatherill M, Scriba TJ. Optimization and interpretation of serial QuantiFERON testing to measure acquisition of mycobacterium tuberculosis infection. Am J Respir Crit Care Med 2017;196:638-648.

36. Brown J, Kumar K, Reading J, Harvey J, Murthy S, Capocci S, Hopkins S, Seneviratne S, Cropley I, Lipman M. Frequency and significance of indeterminate and borderline Quantiferon Gold TB IGRA results. Eur Respir J 2017;50:1701267.

37. Dowdy DW, Golub JE, Chaisson RE, Saraceni V. Heterogeneity in tuberculosis transmission and the role of geographic hotspots in propagating epidemics. Proc Natl Acad Sci 2012;109:9557-9562.

38. Shrestha S, Hill AN, Marks SM, Dowdy DW. Comparing drivers and dynamics of tuberculosis in California, Florida, New York, and Texas. Am J Respir Crit Care Med 2017;196:10501059.

39. Salinas JL, Mindra G, Haddad MB, Pratt R, Price SF, Langer AJ. Leveling of Tuberculosis Incidence - United States, 2013-2015. MMWR Morb Mortal Wkly Rep 2016;65:273-278.

40. Nebenzahl-Guimaraes H, Van Laarhoven A, Farhat MR, Koeken VACM, Mandemakers JJ, Zomer A, Van Hijum SAFT, Netea MG, Murray M, Van Crevel R, Van Soolingen D. Transmissible mycobacterium tuberculosis strains share genetic markers and immune phenotypes. Am J Respir Crit Care Med 2017;195:1519-1527.

41. Farhat MR, Sultana R, Iartchouk O, Bozeman S, Galagan J, Sisk P, Stolte C, NebenzahlGuimaraes H, Jacobson K, Sloutsky A, Kaur D, Posey J, Kreiswirth BN, Kurepina N, Rigouts L, Streicher EM, Victor TC, Warren RM, Van Soolingen D, Murray M. Genetic determinants of drug resistance in mycobacterium tuberculosis and their diagnostic value. Am J Respir Crit Care Med 2016;194:621-630.

42. Haworth CS, Banks J, Capstick T, Fisher AJ, Gorsuch T, Laurenson IF, Leitch A, Loebinger MR, Milburn HJ, Nightingale M, Ormerod P, Shingadia D, Smith D, Whitehead N, Wilson R, Floto RA. British Thoracic Society guidelines for the management of non-tuberculous mycobacterial pulmonary disease (NTM-PD). Thorax 2017;72:ii1-ii64.

43. Novosad SA, Henkle E, Schafer S, Hedberg K, Ku J, Siegel SAR, Choi D, Slatore CG, Winthrop KL. Mortality after Respiratory Isolation of Nontuberculous Mycobacteria. A Comparison of Patients Who Did and Did Not Meet Disease Criteria. Ann Am Thorac Soc 2017;14:1112-1119. 
44. Henkle E, Aksamit T, Barker A, Daley CL, Griffith D, Leitman P, Leitman A, Malanga E, Marras TK, Olivier KN, Prevots DR, Prieto D, Quittner AL, Skach W, Walsh JW, Winthrop KL. Patient-Centered Research Priorities for Pulmonary Nontuberculous Mycobacteria (NTM) Infection. An NTM Research Consortium Workshop Report. Ann Am Thorac Soc 2016;13:S379-84.

45. Colombo RE, Hill SC, Claypool RJ, Holland SM, Olivier KN. Familial clustering of pulmonary nontuberculous mycobacterial disease. Chest 2010;137:629-634.

46. Chen F, Szymanski EP, Olivier KN, Liu X, Tettelin H, Holland SM, Duggal P. Whole-Exome Sequencing Identifies the 6q12-q16 Linkage Region and a Candidate Gene, TTK, for Pulmonary Nontuberculous Mycobacterial Disease. Am J Respir Crit Care Med 2017;196:1599-1604.

47. Olivier KN, Griffith DE, Eagle G, McGinnis JP 2nd, Micioni L, Liu K, Daley CL, Winthrop KL, Ruoss S, Addrizzo-Harris DJ, Flume PA, Dorgan D, Salathe M, Brown-Elliott BA, Gupta R, Wallace RJJ. Randomized Trial of Liposomal Amikacin for Inhalation in Nontuberculous Mycobacterial Lung Disease. Am J Respir Crit Care Med 2017;195:814-823.

48. Satta G, McHugh TD, Mountford J, Abubakar I, Lipman M. Managing pulmonary nontuberculous mycobacterial infection. time for a patient-centered approach. Ann Am Thorac Soc 2014;11:117-121. 